Bamberger finds that, while lardaceous disease of the kidney and other organs was a tolerably frequent attendant on chronic phthisis, the parenchymatous and tubular forms of nephritis were most common. Wishing to investigate this subject from the other standpoint, I endeavoured to ascertain the number of cases of phthisis in which lardaceous disease of the kidney was found after death, and for this purpose examined the records of the Brompton Hospital for one year and a half, during which period Dr. Ewart had been curator, and had examined most of the kidneys microscopically.

In 133 necropsies in cases of phthisis, the kidneys gave a marked reaction with iodine in 50 , or in 37 per cent. In 19 more a slight reaction was obtained, chiefly confined to the Malpighian tufts, giving a total of 69 , or 52 per cent. This is a higher percentage of lardaceous disease in phthisis than any previously given; but it mnst be remembered that the Brompton Hospital has special advantages in point of numbers for investigations of this sort.

The microscopic observations confirm the admirable ones of Dr. Dickinson, demonstrating that the vessels of the Malpighian tufts were those first attacked, and that, later on, the epithelium of the tubuli contorti was involved in the staining. In many instances, fatty changes were also taking place in the epithelium.

Urine.-Having ascertained the pathological conditions of the kidneys, we can better consider the urine secreted by each form. In the lardaceous cases, the average quantity varied from 17 to $40 \mathrm{ozs}$. in the 24 hours. In Case XIV, of which the diagnosis was chronic nephritis, it at one time fell to $2 \frac{1}{2}$ oz., the specific gravity rising to 1047. In this case the urea fell to betwecn 74 and 93 grains, and the patient nevertheless showed no head-symptoms. The specific gravity averages of the lardaceous cases varied from 1012 to 1031, a much higher estimate then Dr. Dickinson's, who gives it as 1006 to 1015. In Case III, where great obstruction occurred in the tubes, it rose gradually from 1014 to 1040 , with such an enormous increase of albumen, that the urine appeared to be a mere solution of serum. In Case XIII, one of granular kidneys, it fell to 1011.

The albumen was very abundant, and seldom amounted to less than half the test-tube. In Case II, the urine eventually became solid on boiling, and prevented further investigations.

In the "granular case," it averaged one-tenth, but never exceeded one-sixth. In Case VII, one of large white kidney, presenting no larda ; zous reaction, the albumen was one-half the test-tube, the specific yravity being only 1012, and the kidneys, after death, weighing $22 \mathrm{oz}$. Casts were found in six cases, granular in two, granular and hyaline in one, hyaline with oil-globules in one, simple hyaline in one, and blood-casts in one.

The urea was determined by Russell and West's method. The maximum in the cases of lardaceous kidney was 337 grains in the 24 hours, which occurred in Case I, the minimum was 54 grains, which occurred in Case II, the normal amount being about 500 grains per diem, showing a great decrease from the ordinary standard.

The most remarkable diminution occurred in Case III, where, in one month, the area diminished from 201 to 54 grains, the quantity of urine remaining the same, and the dietary not greatly varying. In this case, diarrhoa was present, and increased somewhat towards the end of the period; but the increase was not sufficient to reduce the quantity of the urine passed, though probably some of the urea was excreted by the bowels. Our lardaceous cases, therefore, show a decided diminution of water and urea, but the presence of a very large amount of albumen. Unfortunately, time did not allow of the quantitative analysis of the other constituents of the urine in these cases. In column 7 of the Table II, I have entered the rate of urea-excretion per pound of the patient's weight, according to Parkes' plan, who estimated that a healthy man should excrete daily 31 grains for every pound of his weight. This shows that the highest rate was $2 \frac{1}{4}$ grains, and two of the patients excreted 1.6 grains and 1.3 grains per pound respectively, and one rather less than 1 grain per pound-a very small quantity.

The question arises as to the cause of the lowering of the temperature in the above cases, and we naturally turn to the state of the blood for an explanation. In albuminuria, generally, there is a marked decrease in the number of red corpuscles and of albumen, with a relative increase in the water, fibrin, and leucocytes; hence the great pallor and the tendency to dropsy. As the red corpuscles are the carriers of ozygen to the tissues, it would appear that the diminution in their number, which, according to Dickinson, amounts to one-fifth, may considerably interfere with perfect oxidation : but in many cases of disease, pallor is combined with high temperature, and so this will not afford a complete explanation. Nor will the presence of an increased amount of urea alone do so; for it has been shown by Hammond's ${ }^{1}$ important experiments that, provided the kidneys remain intact, a considerable quantity of urea can be injected into the blood of dogs without greatly disturbing the circulation, and that, when symptoms of uræmia appeared, they disappear under the degenerating action of the kidneys. Even when the kidneys have been removed, or the renal arteries tied, and thus the urinary constituents retained within the blood, uræmia does not take place, if, as has been pointed out by Bernard and Barreswil, the urea or the products of its metamorphosis can be discharged by the stomach and intestines. The state of the kidneys in Case II blocked by tubal nephritis, and the small quantity of urea and water excreted by them, without any symptoms of coma, with the obstinate, but doubtless necessary diarrhœa, present a remarkable similarity to this set of experiments. Hammond, however, shows that urea, injected into the blood in quantities beyond such an amount as can be excreted, produces toxic effects.

It is probable, however, that the condition known as uræmia is due to the retention within the blood, not of one urinary constituent alone but of many; and an experiment of Stolnikoff (Centralblatt für die Med. Wiss., April 24th, 1880) bears on this. He found, in experimenting on rabbits, that if, before injecting putrid material into the blood and setting up septicæmia, the renal arteries were compressed, and thus the conditions of nephritis reproduced, no pyrexia followed, but rather a lowering of the temperature. He also found that, when nephrectomy was performed on animals in a feverish state, the fever sank.

The temperature of chronic Bright's disease, whether nephritic or granular, is usually low, and often subnormal ; and so it is not surprising that the state of blood which lowers the temperature in this form of disease should reduce the high temperatures of pyrexial phthisis. We may conclude, therefore, that the lowering of the temperature in cases of phthisis associated with albuminuria is due - partly, at least-to the retention within the blood of the constituents of the urine. This gives rise to uræmia, which is not sufficiently intense to cause coma.

Prognosis.-A careful study of our cases will disclose a very unfavourable outlook for the complication of albuminuria with phthisis. The patients died generally within a few months of the appearance of the first symptoms-one after a few weeks; and the longest case lasted but three months. Private patients, however, appear to live longer, and I know of some cases where life has been prolonged even for years. In the discussion on Dr. Sparks and Dr. Bruce's paper read before the Royal Medical and Chirurgical Society on January 14th, 1879, on the Effect of Diet and Rest in Chronic Nephritis, Dr. Quain cited a case of phthisis of fifteen years' standing, with albuminuria of five years' duration; and one of extreme albuminuria and extensive chronic phthisis, with but little influence on the general health. In one of my patients, who has had phthisis for fifteen years, and a considerable-sized cavity for ten years, albuminuria supervened six years ago, and he still survives, although in a very precarious condition. Another patient, of twelve years' duration, has had slight albuminuria for one year, and is still able to travel, though living the life of an invalid. These are the longest cases $I$ can recall ; and they must be regarded as the exception, and not the rule. Many others whom I have seen have sunk a few months after the albuminuria set in; so that this symptom must, in all cases of phthisis, be regarded as of very serious import.

The treatment of these cases, and the results of various dietetic experiments, are reserved for another communication.

\section{ELECTROLYSIS IN THE TREATMENT OF DRACUNCULUS.}

BY ALEXANDER S. FAULKNER, M.R.C.S.Eng., I.M.D., Civil Surgeon, Aden.

O. L., a Hindu goldsmith from Kattyawar, aged 28, was admitted into the Civil Hospital, Aden, on July 25th last, suffering from the effects of the presence of four Guinea-worms in different parts of his body.

The patient, though a slight man, was fairly nourished, and his general constitution and functions did not seem much impaired. The different positions of the several worms were as follows. About the upper third of the outer aspect of the right leg, a worm had protruded about three inches; on the lower third of the outer side of the left thigh, another had a'so protruded to the extent of about two inches; on the dorsum of the left foot, he had a fistulous open-

\footnotetext{
1 " Uræmi : Intoxication," Am.rican Journal of Medical Sciences, 1861.
} 
ing, indicating the site where a worm appeared, but was broken off in the patient's endeavours to extract it, but the remaining portion of the worm could be distinctly felt in the surrounding subcutaneous tissues ; ard, on the back of the left hand, especially between the little and ring fingers, another worm could not only be felt, but its tortuous course was distinctly visible, but, so far, its presence had caused no inflammation, and, consequently, there was no apparent indication of the site of its intended exit.

The patient had only been in Aden for three months, having previously resided in Kattyawar, in India (a district where this disease is of very common occurrence). The first indication he noticed of his present condition was a "swelling" on the outer aspect of the left thigh, which resulted in an abscess, and subsequently burst, discharging a good deal of pus, and the head of a Guinea-worm. In trying, by forcible traction, to extract the latter, the worm naturally broke, its greater portion remaining within. Subsequently, other abscesses formed, in like manner, in different parts of his body, caused in each case by the presence of a Guinea-worm, all of which were treated by the patient by extracting only portions of a worm at a time. In this way, he stated, he had been suffering for the last five years.

On presenting himself for treatment at the Civil Hospital I determined to try the experiment of applying galvanism to the protruded worms, an idea $I$ have for some time wished to test practically. The result in this case was most encouraging and satisfactory. The patient held one pole of the battery in one of his hands, and the other I applied to the head of the worm which protruded from the patient's right leg; he at once distinctly felt the sensation of the worm "wriggling" within, which caused him slight pain. I left him for about an hour with my hospital-assistant, with instructions that slight and gradual traction should be applied on the worm, and by these means, and in this short space of time, the whole worm was extracted entire. Afterwards, I applied the battery, as before, to the worm protruding from the patient's thigh, and, although the effects on this worm were not quite so satisfactory, still the result was very encouraging, as the entire worm was successfully extracted next day. Poultices were applied over the fistulous opening on the foot and the patient's left hand-the situations of the two other worms. On July 30th four inches of a worm were extracted from the foot after the battery, and subsequently a poultice, had been applied. No other worm reappeared here, and the inflammation soon subsided. I therefore concluded that the whole of the remaining portion of the worm, which the patient had previously broken, had now come away.

On August 3rd, about two inches of the worm on the patient's left hand appeared after the bursting of a small abscess. Galvanism was applied to this worm as in the former instances, and a poultice ordered. Galvanism was again applied on August 5th. The worm had protruded four inches, and was now six inches long. On August 6th the protruded portion of the worm was about twelve inches in length. The battery and a poultice were applied, and in the evening the entire worm was extracted whole. There being now no signs of the presence of any other worms, the patient was discharged from the hospital

REMARKS. - The usual routine-method of treatment in these cases is the application of poultices over any inflammation caused by the presence of a Guinea-worm, and, when once the worm has appeared, means to prevent its re-entry into the body are resorted to, such as twisting the protruded worm round a small quill or other convenient substance, and gradually twisting the worm more and more each day till the whole is extracted, a decidedly lengthened and tedious procedure in most cases, and usually unsatisfactory in its results, as the worm is very apt to be broken, and, if so, the general constitution of the patient suffers. The idea of applying counterirritation to the worm itself to expedite its safe extraction in these cases, first struck me when perusing some notes (Annual Medical Reports of the British Army) by Surgeon Stewart, Bombay Medical Department, in which he stated he had tried the experiment of applying the sting of a scorpion to protruded worms; and my object in recording the above brief notes of $\mathrm{my}$ case is to bring to notice the very satisfactory results I obtained by far simpler and more convenient means of counter-irritation, viz., the application of galvanism to the worm. This no doubt has the effect of paralysing the worm to such an extent that practically there is no resistance to its extraction so far as the worm itself is concerned, and its rapid and entire extraction is consequently made easy if only ordinary care be taken in handling the worm.

Although this mode of treatment is a novel one (so far as I am personally aware), still I feel that the successful result of the above case, obtained by its means, is sufficient in itself to warraxt the hope that others, more favourably situated than myself for meting this disease, will give it a fair trial and record their results.

\section{CLINICAL MEMORANDA.}

\section{A CASE OF ELEPHANTIASIS.}

DURING a holiday, my attention was drawn to the following case in a country village. Several medical men have seen it, and have all been struck with the growth of the legs. A short description of the state of this poor woman may be interesting.

Sarah W., aged 43, was a healthy woman when young. Her family history is good. Fifteen years ago, she was confined of her first child. Labour was natural. All went on right for a few days, when she had a rigor, and the left leg began to swell. The swelling began in the thigh, and spread down to the foot. The skin was erysipelatous. As this limb recovered, the right leg began to be affected in the same way. Both were raised into large blisters below the knee, which discharged a large quantity of serum. The secretion of milk failed for thirteen weeks after the confinement, when the child refused it. She had a large abscess in the left breast, and one in the calf of the left leg. After this, the right leg began to enlarge slowly, and has continued to grow larger for fifteen years. Treatment at first seemed to check it, but only for a time. The left leg soon followed, though it has not attained such a size as the right. There was a miscarriage six months after the confinement. The catamenia have been regular, though scanty. Leucorrhœa is generally present. There is some dysuria, and difficulty in passing urine. The bowels act regularly. She is subject to dyspncea and palpitation. The face looks healthy and natural. The body is very large, but only from adipose tissue. The right leg measures round it, just above the ankle, 35t inches; half-way to the knee it is 39 inches in circumference; while at the top of the calf it is 45 inches. Round the knee-joint it measures 33 inches, and at the middle of the thigh 35 inches. The mass of hypertrophy is divided into lobes by deep fissures, which are smooth at the bottom. The deepest fissure is where the mass overhangs the foot, almost concealing it. It is over three inches in depth, and extends all round the leg. The next is about three inches deep, and almost encircles the limb. A third, 21 inches deep, goes about half-way round the leg. One large lobule, just below the knee on the fibular side, grows out some inches. The latest growth is on the inner surface of the thigh. The fissures have frequently been cracked and ulcerated, with an offensive discharge. The back and lower parts of the leg are rough, like bark, this also partly spreading to the front. Other parts are smooth and glazed. The lobes are firm and dense. The foot is swollen from cedema alone. The left leg is 12 inches round above the ankle, and overhangs the foot; at the calf, it is 26 inches. Save the pain from the cracks when present, and frequent shooting pains in the bowels and legs, the principal suffering is from the size and weight. Mrs. W. can walk about slowly, dragging the right leg. Fuller's earth and liquor carbonis detergens keep it clean and free from smell. She would not allow amputation some years ago, when the left leg was not much affected. Now, nothing but palliative treatment could be carried out. The mass is kept up fairly well by several stockings sewed together. This immense hypertrophy following the puerperal difficulties seems to be worthy of record.

T. E. PURDOM, M.D., C.M., 16, Eldon Square, Newcastle-on-Tyne.

\section{THERAPEUTIC MEMORANDA.}

\section{UNUSUAL SYMPTOMS FOLLOWING APPLICATIONS OF UNGOENTOM HYDRARGYRI AMMONIATI.}

Noticing a paper, by Mr. Green, with the above heading, among the agenda of the Isle of Wight district meeting for October 25th, I send you a brief note of two cases where the application of a precipitated mercury ointment of 30 grains to the ounce, for chronic eczema of the insteps, probably of fungoid origin, was followed by severe and acute symptoms.

CASE I.-Mrs. Z., Eurasian, aged 55, a full-bodied person, applied to me suffering from chronic eczema, over both insteps, of many months' duration. The skin was hypertrophied and discoloured, and the itching caused much annoyance. I prescribed an ointment of 30 grains of hydrargyrum ammoniatum, and 15 minims of compound tincture of lavender, with vaseline, to 